\title{
Generalized Gradient on Vector Bundle - Application to Image Denoising
}

\author{
Thomas Batard and Marcelo Bertalmío * \\ Department of Information and Communication Technologies \\ University Pompeu Fabra, Barcelona, Spain \\ \{thomas . batard, marcelo. bertalmio\}@upf .edu
}

\begin{abstract}
We introduce a gradient operator that generalizes the Euclidean and Riemannian gradients. This operator acts on sections of vector bundles and is determined by three geometric data: a Riemannian metric on the base manifold, a Riemannian metric and a covariant derivative on the vector bundle. Under the assumption that the covariant derivative is compatible with the metric of the vector bundle, we consider the problems of minimizing the L2 and L1 norms of the gradient. In the L2 case, the gradient descent for reaching the solutions is a heat equation of a differential operator of order two called connection Laplacian. We present an application to color image denoising by replacing the regularizing term in the Rudin-Osher-Fatemi (ROF) denoising model by the L1 norm of a generalized gradient associated with a well-chosen covariant derivative. Experiments are validated by computations of the PSNR and Q-index.
\end{abstract}

Keywords: Generalized gradient, Riemannian manifold, Vector bundle, Total variation, Color image denoising, Rudin-Osher-Fatemi model.

\section{Introduction}

Total variation regularization methods have been widely used for image denoising tasks. Given an image $I_{0}: \Omega \subset \mathbb{R}^{2} \longrightarrow \mathbb{R} \in B V(\Omega)$ corrupted by additive white Gaussian noise of standard deviation $\sigma$, the seminal model of Rudin-OsherFatemi (ROF) [19] estimates the denoised image as the solution of the following variational problem

$$
\underset{I \in B V(\Omega)}{\arg \min } \int_{\Omega} \frac{1}{2} \lambda\left(I-I_{0}\right)^{2}+\|\nabla I\| d \Omega
$$

where $\lambda$ is a tuning parameter. The first term in formula (1) is the attached data term and the second one is the regularizing term. Since then, this model has been extended in several ways (see e.g. [4],[9],[14],[15],[16],[17],[18],[24],[25], for local methods based on a modification of the regularizing term, and [8],[10] for nonlocal methods).

\footnotetext{
* This work was supported by European Research Council, grant agreement no. 306337
} 
In this paper, we construct a new regularizing term from a generalization of the Euclidean and Riemannian gradient operators as well as the Jacobian on vector bundles. Then, the ROF denoising model based of this new operator generalizes the Euclidean approach of [19] and its multidimensional extension [4], as well as the Riemannian ROF denoising model in [17]. The key idea is to treat the term $\nabla I$ as a vector-valued differential 1 -form $\nabla^{E} I$, that we call connection gradient of $I$, where the operator $\nabla^{E}$ is a covariant derivative (also called connection). Given Riemannian metrics on the base manifold and vector bundle, a metric on the space of vector-valued differential 1-forms might be constructed, and consequently the norm of the connection gradient $\nabla^{E} I$ might be considered. Then, for particular choices of metrics and covariant derivative, the norm of $\nabla^{E} I$ corresponds to the norm of the Euclidean or Riemannian gradient.

In this paper, we focus on connection gradients where the covariant derivative is compatible with the metric of the vector bundle. In this context, the covariant derivative $\nabla^{E}$ has an adjoint operator $\nabla^{E^{*}}$ and we show that both L1 and L2 norms minimization problems extend the Euclidean and Riemannian approaches in a natural way. Indeed, we show that the gradient descent flow for reaching the sections minimizing the L2 norm of connection gradient is the heat equation of a generalized Laplacian. Moreover, we show that the critical points of the L1 norm of connection gradient satisfy the equation $\nabla^{E^{*}}\left(\nabla^{E} I /\left\|\nabla^{E} I\right\|\right)=0$.

The outline of the paper is the following. Sect. 2 is mainly theoretical. We first introduce the notion of connection gradient and its norm. Then, we restrict to the case where the covariant derivative is compatible with the metric of the vector bundle, and consider the L1 and L2 norms minimization of connection gradient. In Sect. 3, we present an application to color image denoising by considering the L1 norm of a suitable connection gradient as the regularizing term of a ROF denoising model. We test our denoising method on the Kodak database [11] and compute both PSNR and Q-index [23] measures. Results show that our method provides better results than the Split Bregman method [9] applied to ROF functional.

\section{Generalized gradient on vector bundle}

\subsection{Definitions and examples}

We refer to [21] for an introduction to differential geometry of fiber bundles. Given a vector bundle $E$, we denote by $\Gamma(E)$ the set of smooth sections of $E$.

\section{Connection gradient}

Definition 1. Let $E$ be a vector bundle of rank $m$ over a Riemannian manifold $(M, g)$ of dimension $n$. Let $\nabla^{E}$ be a covariant derivative and $h$ be a definite positive metric on $E$. Given $\varphi \in \Gamma(E)$, we call the term $\nabla^{E} \varphi \in \Gamma\left(T^{*} M \otimes E\right)$ the connection gradient of $\varphi$. 
The metrics $g$ on $T M$ and $h$ on $E$ induce a definite positive metric $\langle$,$\rangle on$ $T^{*} M \otimes E$. Then, we define the norm of the connection gradient of $\varphi$ as

$$
\left\|\nabla^{E} \varphi\right\|:=\sqrt{\left\langle\nabla^{E} \varphi, \nabla^{E} \varphi\right\rangle}=\sqrt{\sum_{i, j=1}^{n} g^{i j} h\left(\nabla_{\partial / \partial x_{i}}^{E} \varphi, \nabla_{\partial / \partial x_{j}}^{E} \varphi\right)}
$$

where $\left(\partial / \partial x_{1}, \cdots, \partial / \partial x_{n}\right)$ is the frame of $T M$ induced by a coordinates system $\left(x_{1}, \cdots, x_{n}\right)$ of $M$.

Example 1. Let $E=C^{\infty}(M)$ be the vector bundle of rank 1 of smooth functions on a Riemannian manifold $(M, g)$. Let $\nabla^{E}$ be the trivial covariant derivative on $E$ and $h$ be the definite positive metric on $E$ given by the scalar multiplication. Then, the connection gradient of a function $f$ is its differential $d f \in \Gamma\left(T^{*} M\right)$.

The musical isomorphism $\sharp: T^{*} M \longmapsto T M$ maps $d f$ onto the Riemannian gradient $\nabla_{g} f$ of $f$, of components $g^{i j} \partial f / \partial x_{i}$ in the frame $\left\{\partial / \partial x_{j}\right\}$. Moreover the norm of $d f$ coincides with the norm of $\nabla_{g} f$ since we have

$$
\|d f\|:=\sqrt{\langle d f, d f\rangle}=\sqrt{\sum_{i, j=1}^{n} g^{i j} \frac{\partial f}{\partial x_{i}} \frac{\partial f}{\partial x_{j}}}
$$

\section{Connection compatible with the metric}

Definition 2. Let $E$ be a vector bundle over a Riemannian manifold $(M, g)$, equipped with a definite positive metric $h$. A covariant derivative $\nabla^{E}$ on $E$ is compatible with the metric $h$ if it satisfies

$$
d h(\varphi, \psi)=h\left(\nabla^{E} \varphi, \psi\right)+h\left(\varphi, \nabla^{E} \psi\right)
$$

for any $\varphi, \psi \in \Gamma(E)$.

Example 2. On the vector bundle of smooth functions on a Riemannian manifold, the trivial covariant derivative $\nabla^{E}$ defined by $\nabla^{E} f:=d f$ is compatible with the metric given by the scalar multiplication on the fibers.

Assuming that $E$ is associated with the principal bundle $P_{\mathrm{SO}}(E)$ of orthonormal frame fields of $E$, we have the following result

Proposition 1 (see e.g. Lawson et al. [13] (Prop. 4.4 p.103)). There is a one-one correspondence between connection 1-forms on $P_{S O}(E)$ and covariant derivatives on $E$ that are compatible with the metric.

Under the choice of a local trivializing section of $P_{\mathrm{SO}}(E)$, i.e. a local orthonormal frame with respect to $h$ of the vector bundle $E$, a connection 1-form is a $\mathfrak{s o}(n)$ valued 1-form on $M$, i.e. $\omega \in \Gamma\left(T^{*} M \otimes \mathfrak{s o}(n)\right)$. More precisely, we have

$$
\nabla_{X}^{E} \varphi=d_{X} \varphi+\omega(X)(\varphi)
$$

for any $X \in \Gamma(T M)$. 
Connection Laplacian Let $\nabla^{T^{*} M \otimes E}$ be the covariant derivative on $T^{*} M \otimes E$ defined as

$$
\nabla^{T^{*} M \otimes E}(\eta \otimes \varphi)=\nabla^{T^{*} M} \eta \otimes \varphi+\eta \otimes \nabla^{E} \varphi
$$

where $\nabla^{T^{*} M}$ is the covariant derivative on $T^{*} M$ induced by the Levi-Civita covariant derivative on $(T M, g)$ and $\nabla^{E}$ is a covariant derivative on $E$ compatible with a definite positive metric $h$. The adjoint $\nabla^{E^{*}}: \Gamma\left(T^{*} M \otimes E\right) \longrightarrow \Gamma(E)$ of the operator $\nabla^{E}: \Gamma(E) \longrightarrow \Gamma\left(T^{*} M \otimes E\right)$ is the operator

$$
\nabla^{E^{*}}=-\operatorname{Tr} \nabla^{T^{*} M \otimes E}
$$

where $\operatorname{Tr}$ denotes the contraction with respect to the metric $g$. In others words, the following equality is satisfied

$$
\int_{M} h\left(\nabla^{E^{*}} \eta, \varphi\right) d M=\int_{M}\left\langle\eta, \nabla^{E} \varphi\right\rangle d M
$$

assuming that $\varphi$ has compact support.

Example 3. On the vector bundle of smooth functions on a Riemannian manifold $(M, g)$, the adjoint $d^{*}: \Gamma\left(T^{*} M\right) \longrightarrow C^{\infty}(M)$ of the trivial covariant derivative $d: C^{\infty}(M) \longrightarrow \Gamma\left(T^{*} M\right)$ is the operator

$$
d^{*} \eta=-\sum_{i, j}\left(g^{i j} \partial_{x_{i}} \eta\left(\partial / \partial x_{j}\right)-\sum_{k} \Gamma_{i j}^{k} \eta\left(\partial / \partial x_{k}\right)\right)
$$

where $\Gamma_{i j}^{k}$ are the Christoffel symbols of $(M, g)$.

Definition 3. The connection Laplacian $\Delta^{E}$ is the second order differential operator on $\Gamma(E)$ defined as $\Delta^{E}=\nabla^{E^{*}} \nabla^{E}$.

In the frame $\left(\partial / \partial x_{1}, \cdots, \partial / \partial x_{n}\right)$ of $(T M, g)$, we have

$$
\Delta^{E}=-\sum_{i j} g^{i j}\left(\nabla_{\partial / \partial x_{i}}^{E} \nabla_{\partial / \partial x_{j}}^{E}-\sum_{k} \Gamma_{i j}^{k} \nabla_{\partial / \partial x_{k}}^{E}\right)
$$

Example 4. The Laplace-Beltrami operator is the connection Laplacian (up to a sign) associated to the trivial covariant derivative $d$ on the vector bundle of smooth functions on a Riemannian manifold $(M, g)$, i.e. In other words, it might be written

$$
\Delta^{E}=-\sum_{i j} g^{i j}\left(\partial_{x_{i}} \partial_{x_{j}}-\sum_{k} \Gamma_{i j}^{k} \partial_{x_{k}}\right)
$$

\subsection{L2 minimization of connection gradient and Dirichlet energy}

Let $E$ be a vector bundle over a Riemannian manifold $(M, g)$ equipped with a definite positive metric $h$ and a covariant derivative $\nabla^{E}$ compatible with $h$. We have the following result 
Proposition 2 (Lawson et al. [13] Prop. 8.1 p.154). The operator $\Delta^{E}$ is non-negative and essentially self-adjoint. Furthermore,

$$
\int_{M} h\left(\Delta^{E} \varphi, \psi\right) d M=\int_{M}\left\langle\nabla^{E} \varphi, \nabla^{E} \psi\right\rangle d M
$$

for all $\varphi, \psi \in \Gamma(E)$ provided that one of $\varphi$ or $\psi$ has compact support. If $M$ is compact, then $\Delta^{E} \varphi=0$ if and only if $\nabla^{E} \varphi=0$.

We observe that the right term of equality (8) corresponds to the Gâteaux derivative in the direction $\psi$ of the energy

$$
E(\varphi)=\int_{M}\left\|\nabla^{E} \varphi\right\|^{2} d M
$$

Hence the critical points of the energy (9) satisfy $\Delta^{E} \varphi=0$. Moreover, they correspond to the minimum of the energy.

Example 5. A non trivial covariant derivative $\nabla^{o p t}$ compatible with the metric is constructed in [2] in the context of color image processing. Then, the gradient descent for reaching the sections minimizing the corresponding energy (9) is compared with the Beltrami flow in [20], that may be viewed as the gradient descent for reaching the sections minimizing the energy (9) associated with the trivial covariant derivative. Experiments show that colors and edges and better preserved with the non trivial covariant derivative. The authors explain this behaviour by the fact that the solutions, i.e. the sections $\varphi$ satisfying $\nabla^{o p t} \varphi=0$, are not necessarily constant.

Heat equation and heat kernel of connection Laplacian The gradient descent method for reaching sections minimizing the energy (9) corresponds to the heat equation of the connection Laplacian $\Delta^{E}$

$$
\frac{\partial \varphi}{\partial t}+\Delta^{E} \varphi=0
$$

Results about heat equation and heat kernel of connection Laplacian are welletablished (see e.g. [3]).

Given a connection Laplacian $\Delta^{E}$ and $\varphi_{0} \in \Gamma(E)$, there exists a smooth map called heat kernel of $\Delta^{E}$ and denoted by $K$ such that the operator $e^{-t \Delta^{E}}$ defined by

$$
\left(e^{-t \Delta^{E}} \varphi_{0}\right)(x)=\int_{M} K_{t}(x, y) \varphi_{0}(y) d M
$$

satisfies the heat equation (10).

The heat kernel of a connection Laplacian has a series expansion of the form

$$
\left(\frac{1}{4 \pi t}\right)^{\frac{n}{2}} e^{-d(x, y)^{2} / 4 t} \Psi\left(d(x, y)^{2}\right) \sum_{i=0}^{+\infty} t^{i} \Phi_{i}\left(x, y, \Delta^{E}\right) J(x, y)^{-\frac{1}{2}}
$$


where $\Phi_{i}\left(x, y, \Delta^{E}\right) \in \operatorname{End}\left(E_{y}, E_{x}\right), n$ is the dimension of the base manifold $M$, and $d$ stands for the geodesic distance on $(M, g)$. The function $\Psi$ is such that the term $\Psi\left(d(x, y)^{2}\right)$ equals 1 if $y$ is inside a normal neighborhood of $x$ and 0 otherwise. At last, $J$ are the Jacobians of the coordinates changes from usual coordinates systems to normal coordinates systems.

The leading term of the series (11) is

$$
\left(\frac{1}{4 \pi t}\right)^{n / 2} e^{-d(x, y)^{2} / 4 t} \Psi\left(d(x, y)^{2}\right) \tau(x, y) J(x, y)^{-1 / 2}
$$

where $\tau(x, y)$ is the parallel transport map on $E$ associated to $\nabla^{E}$ along the unique geodesic joining $x$ and $y$.

Example 6. In [22], convolution with the leading term (12) associated to the Laplace-Beltrami operator were applied to anisotropic diffusion of color images. It was extended in [1] to connection Laplacians with no trivial connections.

Parallel section and Harmonic map Harmonic maps between two Riemannian manifolds $\sigma:(M, g) \longrightarrow(N, Q)$ are defined as critical points of the Dirichlet energy

$$
E(\sigma)=\int_{M} \operatorname{Tr}\left(\sigma^{*} h\right) d M=\int_{M}\|d \sigma\|^{2} d M
$$

The Euler-Lagrange equations of the functional (13) are

$$
\tau(\sigma):=\operatorname{Tr} \nabla^{T^{*} M \otimes \sigma^{-1}(T N)} d \sigma=0
$$

Note that the Dirichlet energy (13) is at the core of the Beltrami framework of Sochen et al. (see e.g. [20]).

Theorem 1 (Konderak [12]). Let $E$ be a vector bundle over a compact Riemannian manifold $(M, g)$ equipped with a metric $h$ and a covariant derivative $\nabla^{E}$ compatible with $h$. Let $\tilde{h}$ be the Sasaki metric on $E$ associated to $\left(h, \nabla^{E}, g\right)$. Then $\sigma \in \Gamma(E)$ is a harmonic map $\sigma:(M, g) \longrightarrow(E, \tilde{h})$ if and only if it is parallel, i.e. $\nabla^{E} \sigma=0$.

Hence, the sections minimizing the energy (9) are harmonic maps with respect to the Sasaki metric on the vector bundle.

\subsection{Total Variation on vector bundle}

Definition 4. Let $E$ be a vector bundle over a compact Riemannian manifold $(M, g)$ equipped with a Riemannian metric $h$, and a covariant derivative $\nabla^{E}$ compatible with $h$. We define the total variation $T V$ of $\varphi \in \Gamma(E)$ as

$$
T V(\varphi)=\int_{M}\left\|\nabla^{E} \varphi\right\| d M
$$


Proposition 3. The critical points of (14) are the sections $\varphi$ satisfying

$$
-\operatorname{Tr} \nabla^{T^{*} M \otimes E}\left(\frac{\nabla^{E} \varphi}{\left\|\nabla^{E} \varphi\right\|}\right)=0
$$

Proof. Let $\psi$ be a section with compact support. The first variation of $T V(\varphi)$ in the direction $\psi$ is

$\delta T V(\varphi ; \psi)=\int_{M}\left\langle\frac{\nabla^{E} \varphi}{\left\|\nabla^{E} \varphi\right\|}, \nabla^{E} \psi\right\rangle d M=-\int_{M}\left\langle\operatorname{Tr} \nabla^{T^{M} \otimes E}\left(\frac{\nabla^{E} \varphi}{\left\|\nabla^{E} \varphi\right\|}\right), \psi\right\rangle d M$ since $-\operatorname{Tr} \nabla^{T^{M} \otimes E}$ is the adjoint of $\nabla^{E}$. As $\psi$ has compact support, it follows

$$
\delta T V(\varphi ; \psi)=0 \Longrightarrow-\operatorname{Tr} \nabla^{T^{*} M \otimes E}\left(\frac{\nabla^{E} \varphi}{\left\|\nabla^{E} \varphi\right\|}\right)=0
$$

Remark 1. Formula (15) is not defined where $\nabla^{E} \varphi$ vanishes. One way to tackle this problem is to consider a regularized Total Variation

$$
T V^{r e g}(\varphi)=\int_{M} \sqrt{\left\|\nabla^{E} \varphi\right\|^{2}+\beta} d M, \quad \beta>0
$$

Example 7. Let $E$ be a vector bundle of rank $m$ equipped with the trivial connection and Euclidean scalar product over a compact Euclidean manifold of dimension $n$. Then, for $\varphi \in \Gamma(E)$, we have

$$
T V(\varphi)=\int_{M} \sqrt{\sum_{i=1}^{n} \sum_{j=1}^{m}\left(\frac{\partial \varphi^{j}}{\partial x_{i}}\right)^{2}} d M
$$

Formula (17) corresponds to the total variation defined by Blomgren et al. [4]. In particular, for $n=2$ and $m=1$, this is the total variation of Rudin et al. [19]. Hence, these two approaches may be viewed as Euclidean restrictions of (14).

\section{Application to color image denoising}

\subsection{ROF denoising model on vector bundle: the general case}

Continuous setting Let $E$ be a vector bundle of rank 3 equipped with a definite positive metric $h$ and covariant derivative $\nabla^{E}$ compatible with $h$ over a Riemannian manifold $(M, g)$ of dimension 2. Let $I_{0} \in B V(E)$ be a color image corrupted by additive Gaussian noise of deviation $\sigma$. We propose the denoising model

$$
\underset{I \in B V(E)}{\arg \min } \int_{M} \frac{1}{2} \lambda\left\|I-I_{0}\right\|^{2} d \Omega+\left\|\nabla^{E} I\right\| d M
$$

where $d \Omega$ denotes the Euclidean measure on $M$ and $\lambda$ is a Lagrange multiplier associated with the noise level.

The gradient descent for reaching solutions of (18) is

$$
\frac{\partial I}{\partial t}=-\lambda\left(I-I_{0}\right)+\operatorname{Tr} \nabla^{T^{*} M \otimes E}\left(\frac{\nabla^{E} I}{\left\|\nabla^{E} I\right\|}\right), \quad I_{\mid t=0}=I_{0}
$$


Discrete setting We follow the approach in [19] where forward and backward finite difference operators are used for discretizing the trivial covariant derivative $d$ and its adjoint - div. The key idea is to use the discrete version of the adjoint operator definition, which is written as follows in the context of connection gradient

$$
\int_{M}-\operatorname{div} \eta \varphi d \Omega=\int_{M}\langle\eta, d \varphi\rangle d \Omega
$$

for $\varphi \in C^{\infty}(M)$ and $\eta \in \Gamma\left(T^{*} M\right)$. Then, using forward differences for discretizing $d \varphi$ implies that div $\eta$ must be discretized using backward differences.

We extend this approach by using the general definition of adjoint operator (7). Let us give the explicit expressions in the case of base manifold of dimension 2 and vector bundle of rank 3 . Let $\varphi=\sum_{j=1}^{3} \varphi^{j} e_{j} \in \Gamma(E)$ where $\left(e_{1}, e_{2}, e_{3}\right)$ is orthonormal with respect to $h$. Under the forward finite difference operators for approximating $d \varphi^{j}$, we have

$$
\nabla^{E} \varphi_{m, n}=\sum_{i=1}^{2} \sum_{j=1}^{3}\left[\left(\varphi_{m+\delta_{i 1}, n+\delta_{i 2}}^{j}-\varphi_{m, n}^{j}\right)+\sum_{k=1}^{3} \varphi_{m, n}^{k} \Upsilon_{i k m, n}^{j}\right] d x_{i} \otimes e_{j}
$$

where $\delta$ is the Kronecker symbol. Then, using the discrete form of (7) given by

$$
\sum_{m, n} h_{m, n}\left(\nabla^{E^{*}} \eta_{m, n}, \varphi_{m, n}\right)=\sum_{m, n}\left\langle\eta_{m, n}, \nabla^{E} \varphi_{m, n}\right\rangle_{m, n}
$$

we obtain, for $\eta=\sum_{i=1}^{2} \sum_{j=1}^{3} \eta^{i j} d x_{i} \otimes e_{j}$, the expression $\nabla^{E^{*}} \eta_{m, n}=$

$\sum_{j=1}^{3}\left[\sum_{i, k=1}^{2}\left(g_{m-\delta_{k 1}, n-\delta_{k 2}}^{i k} \eta_{m-\delta_{k 1}, n-\delta_{k 2}}^{i j}-g_{m, n}^{i k} \eta_{m, n}^{i j}\right)+\sum_{r, s=1}^{2} \sum_{p=1}^{3} \eta_{m, n}^{r p} g_{m, n}^{r s} \Upsilon_{s j}^{p}{ }_{m, n}\right] e_{j}$

Hence, as in the Euclidean case [19], forward finite difference operators on $\nabla^{E}$ imply backward finite difference operators on $\nabla^{E^{*}}$.

\subsection{ROF denoising model on vector bundle: an example}

Connection gradient suitable for color image processing Let $I_{0}=\left(I_{0}^{1}, I_{0}^{2}, I_{0}^{3}\right)$ be a color image defined on a domain $\Omega$ of $\mathbb{R}^{2}$.

We construct a surface $S$ embedded in $\left(\mathbb{R}^{5},\|\|_{2}\right)$ parametrized by

$$
\varphi:\left(x_{1}, x_{2}\right) \longmapsto\left(x_{1}, x_{2}, \mu I_{0}^{1}\left(x_{1}, x_{2}\right), \mu I_{0}^{2}\left(x_{1}, x_{2}\right), \mu I_{0}^{3}\left(x_{1}, x_{2}\right)\right), \quad \mu>0
$$

in the fixed orthonormal frame $\left(e_{1}, e_{2}, e_{3}, e_{4}, e_{5}\right)$ of $\left(\mathbb{R}^{5},\|\|_{2}\right)$.

Let $E$ be the vector bundle of $\mathbb{R}^{5}$-valued functions over the Euclidean manifold $\left(\Omega,\|\|_{2}\right)$. Let $\left(Z_{1}, Z_{2}, N_{1}, N_{2}, N_{3}\right)$ be an orthonormal frame field of $E$ with 
respect to the Euclidean norm, where $Z_{1}, Z_{2} \in \Gamma(T S)$. Let $\nabla^{E}$ be the covariant derivative on $E$ given by the connection 1 -form $\omega \equiv 0$ in the frame $\left(Z_{1}, Z_{2}, N_{1}, N_{2}, N_{3}\right)$. Denoting by $P$ the change frame field from $\left(e_{1}, e_{2}, e_{3}, e_{4}, e_{5}\right)$ to $\left(Z_{1}, Z_{2}, N_{1}, N_{2}, N_{3}\right)$, the connection 1-form is given in the frame $\left(e_{1}, e_{2}, e_{3}, e_{4}, e_{5}\right)$ by

$$
P d P^{-1}
$$

As the connection 1-form $\omega$ is $\mathfrak{s o}(\mathfrak{n})$-valued, the covariant derivative $\nabla^{E}$ is compatible with the Euclidean metric on $\mathbb{R}^{5}$.

Remark 2. The orthonormal frame field $\left(Z_{1}, Z_{2}, N_{1}, N_{2}, N_{3}\right)$ of $E$ varies at each point of $\Omega$ since the vector fields $Z_{1}$ and $Z_{2}$ are required to be tangent vector fields of the surface $S$. Moreover, it is not unique since the vector fields $Z_{1}$ and $Z_{2}$ are defined up to rotations in the tangent planes of $S$. By construction, the frame $\left(Z_{1}, Z_{2}, N_{1}, N_{2}, N_{3}\right)$ takes into account the local variations of $I_{0}$.

Algorithm We test the denoising model (18) with this connection gradient.

The algorithm is the following:

1. Consider the (discrete) surface $S$ parametrized by

$$
\varphi:(m, n) \longmapsto\left(m, n, \mu I_{0}^{1}(m, n), \mu I_{0}^{2}(m, n), \mu I_{0}^{3}(m, n)\right), \quad \mu>0
$$

2. Construct an orthonormal moving frame $\left(Z_{1}, Z_{2}, N_{1}, N_{2}, N_{3}\right)$ using GramSchmidt process at each point $(m, n)$ with the assumption that $Z_{1}, Z_{2}(m, n) \in$ $T_{m, n} S$, and denote by $P(m, n)$ the basis change from $\left(e_{1}, e_{2}, e_{3}, e_{4}, e_{5}\right)$ to $\left(Z_{1}, Z_{2}, N_{1}, N_{2}, N_{3}\right)(m, n)$.

3. Embed $I_{0}$ into the frame $\left(e_{1}, e_{2}, e_{3}, e_{4}, e_{5}\right):\left(I_{0}^{1}, I_{0}^{2}, I_{0}^{3}\right) \longrightarrow\left(0,0, I_{0}^{1}, I_{0}^{2}, I_{0}^{3}\right)$.

4. Compute the components of $I_{0}$ in the frame $\left(Z_{1}, Z_{2}, N_{1}, N_{2}, N_{3}\right)$ : $\left(J_{0}^{1}, J_{0}^{2}, J_{0}^{3}, J_{0}^{4}, J_{0}^{5}\right)^{T}:=P^{-1}\left(0,0, I_{0}^{1}, I_{0}^{2}, I_{0}^{3}\right)^{T}$.

5. Perform the Euclidean ROF denoising algorithm on $\left(J_{0}^{1}, J_{0}^{2}, J_{0}^{3}, J_{0}^{4}, J_{0}^{5}\right)^{T}$ with stopping criteria

$$
\frac{1}{|\Omega| \times 3} \sum_{x \in \Omega}\left\|J_{t}(x)-J_{0}(x)\right\|^{2} \geq \sigma^{2}
$$

or

$$
\left|\frac{1}{|\Omega| \times 3} \sum_{x \in \Omega}\left\|J_{t+d t}(x)-J_{0}(x)\right\|^{2}-\frac{1}{|\Omega| \times 3} \sum_{x \in \Omega}\left\|J_{t}(x)-J_{0}(x)\right\|^{2}\right| \leq 0.0005
$$

whichever happens first.

6. Compute the components of the result in the frame $\left(e_{1}, e_{2}, e_{3}, e_{4}, e_{5}\right)$ : $\left(I_{t}^{1}, I_{t}^{2}, I_{t}^{3}, I_{t}^{4}, I_{t}^{5}\right)^{T}:=P\left(J_{t}^{1}, J_{t}^{2}, J_{t}^{3}, J_{t}^{4}, J_{t}^{5}\right)^{T}$, and return the function $\left(I_{t}^{3}, I_{t}^{4}, I_{t}^{5}\right)$.

Experiments We run the algorithm on the Kodak database [11], for $\sigma=$ $5,10,15,20,25$. We take $\mu=0.0075$ for $\sigma=5, \mu=0.005$ for $\sigma=10, \mu=0.0045$ for $\sigma=15, \mu=0.004$ for $\sigma=20$ and $\mu=0.0035$ for $\sigma=25$. The time 
step $d t$ is 0.1 . On Fig. 1, we show that our denoising method removes well the noise. On Fig. 2, we compare our denoising method with the split Bregman denoising method [9] tested online [7]. We observe that our method preserves more the details of the image. We claim that it comes from the choice of the moving frame $\left(Z_{1}, Z_{2}, N_{1}, N_{2}, N_{3}\right)$, that takes into account the local variations of the image. On Fig. 3, we compute the average increases of PSNR as well as the average percent increases of Q-index [23] over the Kodak database for both methods (we define the Q-index of a color image as the mean of the Q-index on each component). We obtain better results with our method.
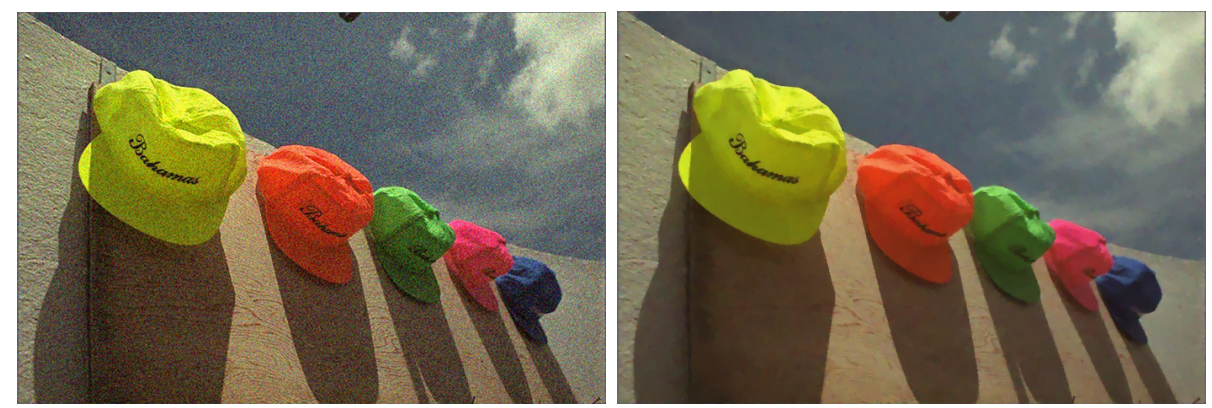

Fig. 1. Example of our denoising method. Left: image corrupted by additive white noise with $\sigma=25$. right: denoised image.
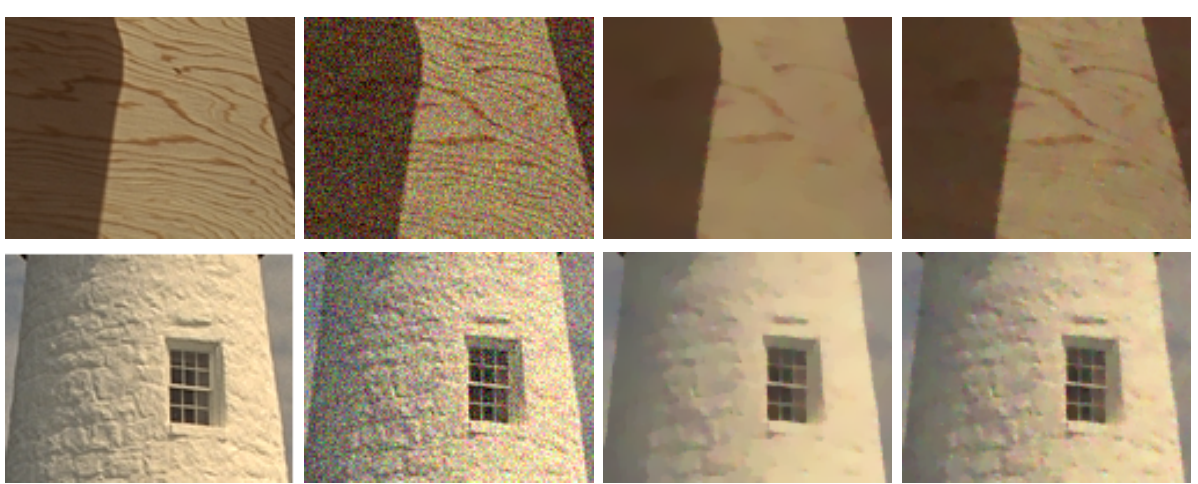

Fig. 2. Comparison of our denoising method with split Bregman: From left to right: original image corrupted by additive white noise with $\sigma=25$, split Bregman denoising method, our denoising method. 

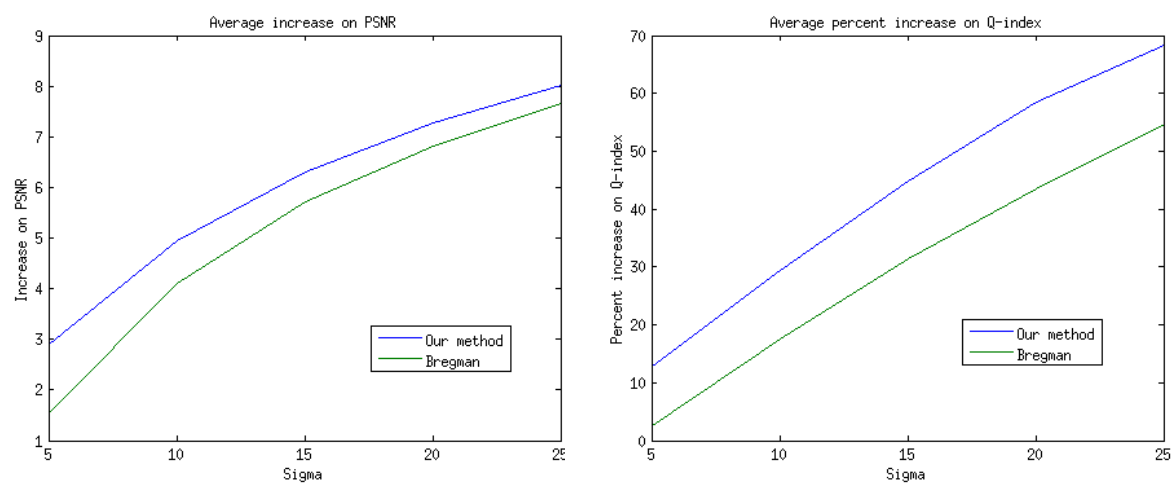

Fig. 3. Comparison of our method with Split Bregman. Left: PSNR increase for each method. Right: Percent increase on Q-index for each method. Values averaged over Kodak database.

\section{Conclusion}

In this paper, we introduced a generalization of the Euclidean and Riemannian gradient operators in the context of vector bundle. We presented an application to image denoising by replacing the Euclidean gradient in the regularizing term of the Rudin-Osher-Fatemi denoising model by a generalized gradient on vector bundle. By the gradient operator we considered, the denoising method is decomposed into 2 steps: first, a projection of the image on the tangent and normal parts of a surface describing the image; then, an Euclidean ROF denoising method of the image in this moving frame. The relevance of the method is justified by the PSNR and Q-index measures. In some sense, the denoising method preserves the first order local geometry of the image.

We would like to point out that the step 2 of our denoising method might be extended to any denoising method. In particular, we expect that nonlocal denoising methods like Non Local Means [5] and BM3D [6] would increase significantly both PSNR and Q-index. More generally, inspired by [8] where the Euclidean ROF model [19] is extended to a nonlocal model by the construction of a nonlocal gradient operator, we expect that our vector bundle ROF model extends to a nonlocal model by the construction of a nonlocal connection gradient operator.

\section{References}

1. Batard, T.: Heat Equations on Vector Bundles - Application to Color Image Regularization. J. Math. Imaging Vis. 41(1-2), 59-85 (2011)

2. Batard, T., Sochen, N.: A Class of Generalized Laplacians Devoted to Multi-Channel Image Processing. To Appear in J. Math. Imaging Vis. DOI 10.1007/s10851-013-0426-7.

3. Berline, N., Getzler, E., Vergne, M.: Heat Kernels and Dirac Operators. Springer (2004) 
4. Blomgren, P., Chan, T.F.: Color TV: Total Variation Methods for Restoration of Vector-Valued Images. IEEE Trans. Image Processing 7(3), 304-309 (1998)

5. Buades, A., Coll, B., Morel, J.-M.: A Review of Image Denoising Algorithms, with a new one. Multiscale Model. Simul. 4(2), 490-530 (2005)

6. Dabov, K., Foi, V., Katkovnik, V., Egiazarian, K.: Image Denoising by Sparse 3-D Transform-Domain Collaborative Filtering. IEEE Trans. Image Processing 16(8), 2080-2095 (2007)

7. Getreuer, P.: Rudin-Osher-Fatemi Total Variation Denoising using Split Bregman. Image Processing On Line (2012)

8. Gilboa, G., Osher, S.: Nonlocal Operators with Applications to Image Processing. Multiscale Model. Simul. 7(3), 1005-1028 (2008)

9. Goldstein, T., Osher, S.: The Split Bregman Method for L1 Regularized Problems. SIAM J. Imaging Sciences 2, 323-343 (2009)

10. Jin, Y., Jost, J., Wang, G.: A New Nonlocal $H^{1}$ Model for Image Denoising. To Appear in J. Math. Imaging Vis. DOI 10.1007/s10851-012-0395-2

11. Kodak, http://rOk.us/graphics/kodak/

12. Konderak, J.J.: On Sections of Fiber Bundles which are Harmonic Maps. Bull. Math. Soc. Sci. Math. Roumanie (4) 90, 341-352 (1999)

13. Lawson, H.B., Michelson, M-L.: Spin Geometry. Princeton University Press (1989)

14. Lysaker, M., Osher, S., Tai, X.-C.: Noise Removal using Smoothed Normals and Surface Fitting. IEEE Trans. Image Processing 13(10), 1345-1357 (2004)

15. Osher, S., Burger, M., Goldfarb, D., Xu, J., Yin, W.: An Iterative Regularization Method for Total Variation-based Image Restoration. Multiscale Model. Simul. 4(2), 460-489 (2004)

16. Rahman, T., Tai, X.-C., Osher, S.: A TV-Stokes Denoising Algorithm. SSVM, ser. Lecture Notes in Computer Science, Sgallari, F., Murli, A., Paragios, N. Eds 4485, 473-483 (2007)

17. Rosman, G., Tai, X.-C., Dascal, L., Kimmel, R.: Polyakov Action Minimization for Efficient Color Image Processing. ECCV Workshops (1), LNCS, vol. 6554, 50-61 (2010)

18. Rosman, G., Wang, Y., Tai, X.-C., Kimmel, R., Bruckstein, A.M.: Fast Regularization of Matrix-Valued Images. ECCV (3), LNCS, vol. 7574, 173-186 (2012)

19. Rudin, L.I., Osher, S., Fatemi, E.: Nonlinear Total Variation Based Noise Removal Algorithms. Physica D 60, 259-268 (1992)

20. Sochen, N., Kimmel, R., Malladi, R.: A General Framework for Low Level Vision. IEEE Trans. Image Processing 7(3), 310-318 (1998)

21. Spivak, M.: A Comprehensive Introduction to Differential Geometry. Publish or Perish, 2nd edition (1990)

22. Spira, A., Kimmel, R., Sochen, N.: A Short-time Beltrami Kernel for Smoothing Images and Manifolds. IEEE Trans. Image Processing 16, 1628-1636 (2007)

23. Wang, Z., Bovik, A.: A Universal Image Quality Index. IEEE Signal Processing Letters 9(3), 81-84 (2002)

24. Weickert, J., Brox., T.: Diffusion and Regularization of Vector- and Matrix-Valued Images. In Nashed, M.Z., Scherzer, O. (eds.) Inverse Problems, Image Analysis, and Medical Imaging. Contemporary Mathematics, vol. 313, 251-268. AMS, Providence (2002)

25. Zhu, W., Chan, T.F.: Image Denoising using Mean Curvature of Image Surface. SIAM J. Imaging Sciences 5(1), 1-32 (2012) 